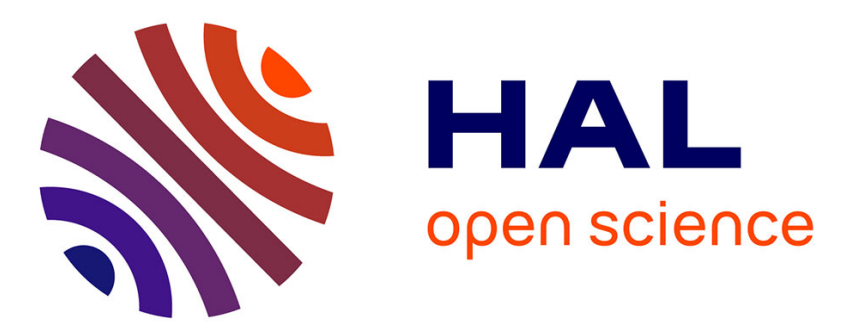

\title{
Nonconforming finite element approximation of the Giesekus model for polymer flows
}

\author{
Roland Becker, Daniela Capatina, Didier Graebling, Julie Joie
}

\section{To cite this version:}

Roland Becker, Daniela Capatina, Didier Graebling, Julie Joie. Nonconforming finite element approximation of the Giesekus model for polymer flows. Computers and Fluids, 2011, 46 (1), pp.142 - 147. 10.1016/j.compfluid.2011.01.036 . hal-03482680

\section{HAL Id: hal-03482680 \\ https://hal.science/hal-03482680}

Submitted on 16 Dec 2021

HAL is a multi-disciplinary open access archive for the deposit and dissemination of scientific research documents, whether they are published or not. The documents may come from teaching and research institutions in France or abroad, or from public or private research centers.
L'archive ouverte pluridisciplinaire HAL, est destinée au dépôt et à la diffusion de documents scientifiques de niveau recherche, publiés ou non, émanant des établissements d'enseignement et de recherche français ou étrangers, des laboratoires publics ou privés. 


\title{
Nonconforming finite element approximation of the Giesekus model for polymer flows
}

\author{
R. Becker ${ }^{\mathrm{a}}$, D. Capatina ${ }^{\mathrm{a}}$, D. Graebling ${ }^{\mathrm{b}}$, J. Joie J,a $^{*}$ \\ ${ }^{a}$ INRIA Bordeaux-Sud Ouest \& LMA UMR CNRS 5142, Université de Pau et des Pays de l'Adour \\ Bât IPRA, Avenue de l'université, 64013 PAU Cédex \\ ${ }^{b}$ INRIA Bordeaux-Sud Ouest \& I.P.R.E.M / E.P.C.P., Université de Pau et des Pays de l'Adour \\ 2, avenue du Président Angot, 64053 PAU Cédex 09
}

\begin{abstract}
We present a numerical approximation of the Giesekus equation which is considered as a realistic model for polymer flows. We use nonconforming finite elements on quadrilateral grids which necessitate the addition of two stabilization terms. An appropriate upwind scheme is employed for the convective term. The underlying discrete Stokes problem is then analysed. Finally, numerical tests are presented in order to validate the code, illustrating its good behavior for large Weissenberg numbers. Comparisons with Polyflow ${ }^{\circledR}$ and with the literature are also carried out.
\end{abstract}

Key words: Polymer liquids, Giesekus model, nonconforming finite elements, high Weissenberg number

\section{INTRODUCTION}

We are interested in the numerical simulation of polymeric liquids which are, from a rheological point of view, non-Newtonian viscoelastic fluids. Their viscoelastic behavior can be observed in a variety of physical phenomena, such as die swelling or the Weissenberg effect, which are unseen with Newtonian liquids and which cannot be predicted by the Navier-Stokes equations.

Despite numerous efforts, the numerical approximation of polymer flows is still a challenging research area, due to the internal coupling between the viscoelasticity of the liquid and the flow, which is quantified by the Weissenberg number $W e=\lambda \dot{\gamma}$ with $\dot{\gamma}$ the shear rate and $\lambda$ the relaxation time.

A major issue to be addressed is the breakdown in convergence of the algorithms at critical values of $W e$. The existing commercial codes are generally only able to deal with $W e$ up to 10 , which is insufficient to describe polymer flows in a processing machine.

The rheological behavior of polymers is so complex that many different constitutive equations have been proposed in the literature in order to describe these phenomena, see for instance [13]. We choose here to study the differential model of Giesekus which presents two main advantages. First, it yields a realistic behavior for shear flows, elongational flows and mixed flows. Second, only two material parameters (the viscosity $\eta$ and the relaxation time $\lambda$ )

\footnotetext{
*I thank ESF for its support to attend to ICFD 2010.

Email addresses: roland.becker@univ-pau.fr (R. Becker),

daniela.capatina@univ-pau.fr (D. Capatina),

didier.graebling@univ-pau.fr (D. Graebling),

julie.joie@univ-pau.fr (J. Joie)
}

are needed to describe the model. However, the Giesekus constitutive law is strongly nonlinear since it involves a quadratic term in the stress tensor.

Our goal is to develop a robust numerical scheme to obtain realistic simulation for high Weissenberg numbers. We consider here the 2D steady case and quadrilateral meshes. We approximate the velocity and the pressure by means of nonconforming finite elements of RannacherTurek, which are well-known to be inf-sup stable, and the stress tensor by means of totally discontinuous piecewise functions. The analysis of the underlying discrete Stokes problem has highlighted the necessity of adding two stabilization terms, one in order to recover a Korn type inequality on nonconforming spaces, and the other to attain optimal convergence. Concerning the Giesekus equation, the convective term on the stress tensor is treated using an upwind scheme, similarly to the well-known LesaintRaviart scheme.

The paper is organized as follows. In Section 2 we introduce the Giesekus model. In Section 3, we describe the numerical scheme and we perform the numerical analysis of the underlying Stokes problem. In particular the influence of the regularization terms is discussed. The last section is devoted to the numerical results. We first study the convergence rate for the Giesekus model on an academic test-case. Then we consider a benchmark problem, the flow past a cylinder, for which we carry out some comparisons and we illustrate the good behavior of the method for large Weissenberg numbers. The robustness of the scheme is explained by the positive definiteness of the conformation tensor, guaranteed by our choice of discretization. 


\section{THE GIESEKUS MODEL}

In what follows, we write the vectors in bold letters and the second order tensors in underlined letters.

Giesekus introduced in [6] the following constitutive law, describing the behavior of a polymeric liquid in a polygonal domain $\Omega \subset \mathbb{R}^{2}$ :

$$
\lambda\left(\underline{\nabla}+\frac{\alpha}{\eta} \underline{\tau} \underline{\tau}\right)+\underline{\tau}=2 \eta \underline{D}(\boldsymbol{u})
$$

with $\underline{\tau}$ the viscous stress tensor, $\underline{D}(\boldsymbol{u})=\frac{1}{2}\left(\underline{\nabla} \boldsymbol{u}+\underline{\nabla} \boldsymbol{u}^{T}\right)$ the strain rate tensor and $\alpha \in] 0,1[$ a parameter. We take $\alpha=0.5$ which seems to be an appropriate choice. Here above, $\nabla \boldsymbol{u}=\left(\frac{\partial u_{i}}{\partial x_{j}}\right)_{1 \leq i, j \leq 2}$ and $\stackrel{\nabla}{\underline{\tau}}$ is the upper convective derivative, defined in the steady case by:

$$
\stackrel{\nabla}{\tau}=(\boldsymbol{u} \cdot \nabla) \underline{\tau}-\underline{\tau} \nabla \boldsymbol{u}^{T}-\nabla \boldsymbol{u} \underline{\tau} .
$$

The complete Giesekus model is obtained by adding the mass and the momentum conservation laws, where the density $\rho$ is supposed to be constant:

$$
\begin{aligned}
\nabla \cdot \boldsymbol{u} & =0, \\
\rho(\boldsymbol{u} \cdot \nabla) \boldsymbol{u}-\nabla \cdot \underline{\tau}+\nabla p & =\boldsymbol{f},
\end{aligned}
$$

and boundary conditions $\boldsymbol{u}=\boldsymbol{g}$ on $\partial \Omega, \underline{\tau}=\underline{\tau}^{D}$ on the inflow boundary $\partial \Omega^{-}=\{x \in \partial \Omega ; \boldsymbol{u}(x) \cdot \boldsymbol{n}(x)<0\}$. Other boundary conditions for $\boldsymbol{u}$ will be considered in subsection 4.2. We take $\boldsymbol{f} \in\left(L^{2}(\Omega)\right)^{2}, \boldsymbol{g} \in\left(H^{1 / 2}(\partial \Omega)\right)^{2}$ and $\underline{\tau}^{D} \in \underline{L}_{\text {sym }}^{2}\left(\partial \Omega^{-}\right)$, with:

$$
\underline{L}_{\text {sym }}^{2}(\omega)=\left\{\underline{\tau}=\left(\tau_{i j}\right)_{1 \leq i, j \leq 2} ; \underline{\tau}=\underline{\tau}^{T}, \tau_{i j} \in L_{2}(\omega)\right\} .
$$

\section{FINITE ELEMENT APPROXIMATION}

\subsection{Discrete nonlinear formulation}

Let $\left(\mathcal{K}_{h}\right)_{h>0}$ be a family of regular meshes of $\Omega$ consisting of quadrilaterals: $\bar{\Omega}=\bigcup_{K \in \mathcal{K}_{h}} K$. We denote by $\varepsilon_{h}^{\text {int }}$ the set of internal edges of $\mathcal{K}_{h}$, by $\varepsilon_{h}^{\partial}$ the set of boundary edges and we put $\varepsilon_{h}=\varepsilon_{h}^{i n t} \cup \varepsilon_{h}^{\partial}$. As usually, let $h_{K}$ be the diameter of the quadrilateral $K$ and let $h=\max _{K \in \mathcal{K}_{h}} h_{K}$.

On every edge $e$ belonging to $\varepsilon_{h}^{i n t}$, such that $\{e\}=$ $\partial K_{1} \cap \partial K_{2}$, we define once and for all a unit normal $\boldsymbol{n}_{e}$. For a given function $\varphi$ with $\varphi_{\mid K_{i}} \in \mathcal{C}\left(K_{i}\right)(1 \leq i \leq 2)$, we define on $e: \varphi^{i n}(\boldsymbol{x})=\lim _{\varepsilon \backslash 0} \varphi\left(\boldsymbol{x}-\varepsilon \boldsymbol{n}_{e}\right), \varphi^{e x}(\boldsymbol{x})=$ $\lim _{\varepsilon \searrow 0} \varphi\left(\boldsymbol{x}+\varepsilon \boldsymbol{n}_{e}\right)$ as well as the jump $[\varphi]=\varphi^{i n}-\varphi^{e x}$ and the average $\{\varphi\}=\frac{1}{2}\left(\varphi^{i n}+\varphi^{e x}\right)$. If $e \in \varepsilon_{h}^{\partial}, \boldsymbol{n}$ is the outward unit normal and $[\varphi]$ is the trace of $\varphi$. We agree to denote the $L^{2}(\omega)$-orthogonal projection of a given function $\varphi \in L^{2}(\omega)$ on the polynomial space $P_{k}(k \in \mathbb{N})$ by $\pi_{k}^{\omega} \varphi$. As usually, we denote by $\varphi^{-}=\min \{0, \varphi\}$ the negative part of $\varphi$ and we set $\varphi^{+}=\varphi-\varphi^{-}$. We denote by $c$ any constant independent of $h, \eta$ and the stabilization parameters. We shall use the notation $\underline{\tau}: \underline{\theta}=\sum_{i, j=1}^{2} \tau_{i j} \theta_{i j}$.

We approach the velocity by nonconforming finite elements of Rannacher-Turek (see [14]) whose degrees of freedom are the mean values across the edges, and the pressure and the stress tensor by totally discontinuous piecewise functions. Let $\hat{K}=[-1,1] \times[-1,1], \Psi_{K}$ : $\hat{K} \rightarrow K$ the bilinear one-to-one transformation and $\hat{Q}_{1}^{r o t}=$ $\operatorname{vect}\left\{1, \hat{x}, \hat{y}, \hat{x}^{2}-\hat{y}^{2}\right\}$. Then we define the space $Q_{K}=$ $\left\{v ; v \circ \Psi_{K} \in \hat{Q}_{1}^{r o t}\right\}$ and we introduce the discrete spaces:

$$
\begin{gathered}
\boldsymbol{V}_{h}=\left\{\boldsymbol{v}_{h} \in\left(L^{2}(\Omega)\right)^{2} ; \boldsymbol{v}_{h \mid K} \in\left(Q_{K}\right)^{2} \forall K \in \mathcal{K}_{h},\right. \\
\left.\frac{1}{|e|} \int_{e}\left[\boldsymbol{v}_{h}\right] d s=0 \forall e \in \varepsilon_{h}^{i n t}\right\}, \\
\boldsymbol{V}_{h}^{\boldsymbol{g}}=\left\{\boldsymbol{v}_{h} \in \boldsymbol{V}_{h} ; \int_{e} \boldsymbol{v}_{h} d s=\int_{e} \boldsymbol{g} d s \forall e \in \varepsilon_{h}^{\partial}\right\}, \\
Q_{h}=\left\{q_{h} \in L_{0}^{2}(\Omega) ; q_{h \mid K} \in P_{0} \forall K \in \mathcal{K}_{h}\right\}, \\
\underline{X}_{h}=\left\{\underline{\theta}_{h} \in \underline{L}_{s y m}^{2}(\Omega) ;\left(\underline{\theta}_{h}\right)_{\mid K} \in \underline{P}_{0} \forall K \in \mathcal{K}_{h}\right\} .
\end{gathered}
$$

We consider the following discrete formulation:

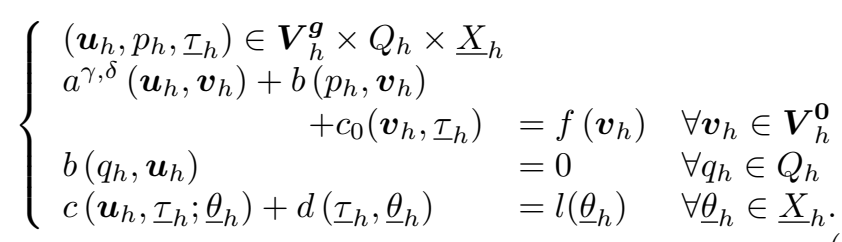

The previous forms are defined by:

$$
\begin{aligned}
a^{\gamma, \delta}(\cdot, \cdot) & =a_{0}(\cdot, \cdot)+\gamma J(\cdot, \cdot)+\delta R(\cdot, \cdot), \\
b\left(q_{h}, \boldsymbol{v}_{h}\right) & =-\sum_{K \in \mathcal{K}_{h}} \int_{K} q_{h} \nabla \cdot \boldsymbol{v}_{h} d x, \\
c(\cdot, \cdot ; \cdot) & =-2 \eta c_{0}(\cdot, \cdot)+c_{1}(\cdot, \cdot ; \cdot)-c_{2}(\cdot, \cdot ; \cdot), \\
d(\cdot, \cdot) & =d_{0}(\cdot, \cdot)+d_{1}(\cdot, \cdot), \\
f\left(\boldsymbol{v}_{h}\right) & =\sum_{K \in \mathcal{K}_{h}} \int_{K} \boldsymbol{f} \cdot \boldsymbol{v}_{h} d x, \\
l\left(\underline{\theta}_{h}\right) & =-\sum_{e \in \varepsilon_{h}^{\partial} \cap \partial \Omega^{-}} \int_{e}\left(\boldsymbol{u}_{h} \cdot \boldsymbol{n}\right)^{-} \underline{\tau}^{D}: \underline{\theta}_{h} d s,
\end{aligned}
$$

where

$$
\begin{aligned}
c_{0}\left(\underline{\tau}_{h}, \boldsymbol{v}_{h}\right) & =\sum_{K \in \mathcal{K}_{h}} \int_{K} \underline{\tau}_{h}: \underline{D}\left(\boldsymbol{v}_{h}\right) d x, \\
c_{2}\left(\boldsymbol{u}_{h}, \underline{\tau}_{h} ; \underline{\theta}_{h}\right) & =\lambda \sum_{K \in \mathcal{K}_{h}} \int_{K}\left(\underline{\tau}_{h} \underline{\nabla} \boldsymbol{u}_{h}^{T}+\underline{\nabla} \boldsymbol{u}_{h} \underline{\tau}_{h}\right): \underline{\theta}_{h} d x, \\
d_{0}\left(\underline{\theta}_{h}, \underline{\tau}_{h}\right) & =\sum_{K \in \mathcal{K}_{h}} \int_{K} \underline{\theta}_{h}: \underline{\tau}_{h} d x \\
d_{1}\left(\underline{\tau}_{h}, \underline{\theta}_{h}\right) & =\frac{\lambda}{2 \eta} \sum_{K \in \mathcal{K}_{h}} \int_{K}\left(\underline{\tau}_{h} \underline{\tau}_{h}\right): \underline{\theta}_{h} d x .
\end{aligned}
$$

The form $c_{1}(\cdot, \cdot ; \cdot)$ approximates the convective term $\boldsymbol{u} \cdot \nabla \underline{\tau}$. We extend the approach of Lesaint-Raviart [10] for constant vectors $\boldsymbol{u}$ to the present nonconforming approximation of the velocity. Thus we approach $\int_{\Omega} \boldsymbol{u} \cdot \nabla \underline{\tau}: \underline{\theta} d x$ by $-\sum_{e \in \varepsilon_{h}} \int_{e}\left\{\boldsymbol{u}_{h} \cdot \boldsymbol{n}_{e}\right\}^{-}\left[\underline{\tau}_{h}\right]: \underline{\theta}_{h}^{i n} d s$. Finally, an integration 
by parts together with the fact that $\pi_{0}^{K} \nabla \cdot \boldsymbol{u}_{h}=0$ for any $K \in \mathcal{K}_{h}$ allow us to write the previous term as follows:

$$
c_{1}\left(\boldsymbol{u}_{h}, \underline{\tau}_{h} ; \underline{\theta}_{h}\right)=\lambda \sum_{e \in \varepsilon_{h}} \int_{e} F_{e}\left(\underline{\tau}_{h}, \boldsymbol{u}_{h}, \boldsymbol{n}_{e}\right):\left[\underline{\theta}_{h}\right] d s,
$$

where $F_{e}\left(\underline{\tau}_{h}, \boldsymbol{u}_{h}, \boldsymbol{n}_{e}\right)=\left\{\boldsymbol{u}_{h} \cdot \boldsymbol{n}_{e}\right\}^{+} \underline{\tau}_{h}^{\text {in }}+\left\{\boldsymbol{u}_{h} \cdot \boldsymbol{n}_{e}\right\}^{-} \underline{\tau}_{h}^{\mathrm{ex}}$ is the numerical flux. We take :

$a_{0}\left(\boldsymbol{u}_{h}, \boldsymbol{v}_{h}\right)=\sum_{K \in \mathcal{K}_{h}} \int_{K} \frac{\rho}{2}\left(\boldsymbol{u}_{h} \cdot \underline{\nabla} \boldsymbol{u}_{h} \cdot \boldsymbol{v}_{h}-\boldsymbol{u}_{h} \cdot \underline{\nabla} \boldsymbol{v}_{h} \cdot \boldsymbol{u}_{h}\right) d x$

The additional forms $J(\cdot, \cdot)$ and $R(\cdot, \cdot)$ ensure the discrete coercivity and are defined by:

$$
\begin{array}{r}
J\left(\boldsymbol{u}_{h}, \boldsymbol{v}_{h}\right)=\eta \sum_{e \in \varepsilon_{h}^{\text {int }}} \frac{1}{|e|} \int_{e}\left[\pi_{1}^{e}\left(\boldsymbol{u}_{h} \cdot \boldsymbol{n}_{e}\right)\right]\left[\pi_{1}^{e}\left(\boldsymbol{v}_{h} \cdot \boldsymbol{n}_{e}\right)\right] d s, \\
R\left(\boldsymbol{u}_{h}, \boldsymbol{v}_{h}\right)=\eta \sum_{K \in \mathcal{K}_{h}} \int_{K}\left(\underline{D}\left(\boldsymbol{u}_{h}\right)-\pi_{0}^{K} \underline{D}\left(\boldsymbol{u}_{h}\right)\right): \underline{D}\left(\boldsymbol{v}_{h}\right) d x .
\end{array}
$$

The stabilization parameters $\gamma, \delta$ are independent of $h$.

Another possibility for the approximation of viscoelastic flows is to introduce the strain rate tensor $\underline{d}=\underline{D}(\boldsymbol{u})$ as a fourth unknown and to split the stress tensor $\underline{\tau}$ (see [7] for the DEVSS method). Then the elimination of $\underline{d}$ at the discrete level yields a three-fields formulation with an additional term similar to our regularization term $R(\cdot, \cdot)$.

The nonlinear problem (2) is solved by Newton's method.

\subsection{Influence of the stabilization terms}

In order to highlight the necessity of adding these regularization terms, we consider in what follows the Stokes equations for a Newtonian liquid $(\lambda=0$ and $\rho=0)$. We can then recover the stress tensor by $\underline{\tau}_{{ }_{\mid K}}=2 \eta \pi_{0}^{K} \underline{D}\left(\boldsymbol{u}_{h}\right)$ and obtain the following two-fields formulation:

$$
\left\{\begin{array}{ccc}
\left(\boldsymbol{u}_{h}, p_{h}\right) \in \boldsymbol{V}_{h}^{\boldsymbol{g}} \times Q_{h} & \\
\tilde{a}^{\gamma, \delta}\left(\boldsymbol{u}_{h}, \boldsymbol{v}_{h}\right)+b\left(p_{h}, \boldsymbol{v}_{h}\right) & =l\left(\boldsymbol{v}_{h}\right) & \forall \boldsymbol{v}_{h} \in \boldsymbol{V}_{h}^{\mathbf{0}} \\
b\left(q_{h}, \boldsymbol{u}_{h}\right) & =0 & \forall q_{h} \in Q_{h}
\end{array}\right.
$$

where

$$
\begin{aligned}
\tilde{a}^{\gamma, \delta}(\cdot, \cdot) & =e(\cdot, \cdot)+\gamma J(\cdot, \cdot)+\delta R(\cdot, \cdot) \\
e\left(\boldsymbol{u}_{h}, \boldsymbol{v}_{h}\right) & =2 \eta \sum_{K \in \mathcal{K}_{h}} \int_{K} \pi_{0}^{K} \underline{D}\left(\boldsymbol{u}_{h}\right): \pi_{0}^{K} \underline{D}\left(\boldsymbol{v}_{h}\right) d x .
\end{aligned}
$$

It is useful to introduce the semi-norm on $\boldsymbol{H}^{1}(\Omega)+\boldsymbol{V}_{h}$ :

$$
[[\boldsymbol{v}]]=\left(2 \eta \sum_{K \in \mathcal{K}_{h}}\|\underline{D}(\boldsymbol{v})\|_{0, K}^{2}+\gamma J(\boldsymbol{v}, \boldsymbol{v})\right)^{1 / 2}
$$

which is a norm on $\boldsymbol{V}_{h}^{\mathbf{0}}$. In order to recover a Korn type inequality on the nonconforming space $\boldsymbol{V}_{h}^{\mathbf{0}}$, we assume that the transformation $\Psi_{K}$ is affine for all $K \in \mathcal{K}_{h}$ and we apply then a result of [2] for piecewise $H^{1}$ functions, improved in [11]:
$\sum_{K \in \mathcal{K}_{h}}\|\boldsymbol{v}\|_{1, K}^{2} \leq c\left(\sum_{K \in \mathcal{K}_{h}}\|\underline{D}(\boldsymbol{v})\|_{0, K}^{2}+\frac{1}{\eta} J(\boldsymbol{v}, \boldsymbol{v})+\phi^{2}(\boldsymbol{v})\right)$,

where $\phi: \boldsymbol{H}^{1}(\Omega) \rightarrow \mathbb{R}$ is a semi-norm such that if $\phi(\boldsymbol{v})=0$ for a rigid motion $\boldsymbol{v}$, then $\boldsymbol{v}$ is constant. By taking $\phi(\boldsymbol{v})^{2}=$ $\sum_{e \in \varepsilon_{h}^{\partial}}\left\|\pi_{0} \boldsymbol{v}\right\|_{0, e}^{2}$ which vanishes on $\boldsymbol{V}_{h}^{\mathbf{0}}$, one obtains :

$$
\sum_{K \in \mathcal{K}_{h}}\|\boldsymbol{v}\|_{1, K}^{2} \leq c\left(\sum_{K \in \mathcal{K}_{h}}\|\underline{D}(\boldsymbol{v})\|_{0, K}^{2}+\frac{1}{\eta} J(\boldsymbol{v}, \boldsymbol{v})\right), \forall \boldsymbol{v} \in \boldsymbol{V}_{h}^{\mathbf{0}}
$$

Theorem 1. Problem (3) has a unique solution.

Proof. We check the discrete hypotheses of the BabuškaBrezzi theorem with respect to the norms $[[\cdot]]$ and $\|\cdot\|_{0, \Omega}$ on $\boldsymbol{V}_{h}^{\mathbf{0}}$ and $Q_{h}$. The proof of the uniform coercivity (w.r.t. $h$ and $\gamma)$ of $a_{h}^{\gamma, \delta}(\cdot, \cdot)$ on Kerb $b_{h}$ is classical. For $\delta \leq 2$ the coercivity constant is also independent of $\delta$. For the discrete inf-sup condition, we use the continuous one, the nonconforming interpolation operator and a trace inequality to bound the term $J(\cdot, \cdot)$.

We have obtained optimal a priori error bounds:

Theorem 2. Let $(\boldsymbol{u}, p) \in \boldsymbol{H}^{2}(\Omega) \times H^{1}(\Omega)$ be the solution of the continuous Stokes problem. Then the solution $\left(\boldsymbol{u}_{h}, p_{h}\right)$ of (3) satisfies:

$$
\left[\left[\boldsymbol{u}-\boldsymbol{u}_{h}\right]\right]+\frac{1}{\sqrt{\eta}}\left\|p-p_{h}\right\|_{0, \Omega} \leq \operatorname{ch}\left(\sqrt{\eta}|\boldsymbol{u}|_{2, \Omega}+\frac{1}{\sqrt{\eta}}|p|_{1, \Omega}\right) .
$$

Proof. The solution $(\boldsymbol{u}, p)$ satisfies the following consistency result, for all $\boldsymbol{v}_{h} \in \boldsymbol{V}_{h}^{\mathbf{0}}$ :

$$
\begin{aligned}
a_{h}^{\delta}\left(\boldsymbol{u}, \boldsymbol{v}_{h}\right)+b\left(p, \boldsymbol{v}_{h}\right) & =f\left(\boldsymbol{v}_{h}\right)+\Sigma_{p}\left(p, \boldsymbol{v}_{h}\right) \\
& +\Sigma_{u}\left(\boldsymbol{u}, \boldsymbol{v}_{h}\right)+\varepsilon\left(\boldsymbol{u}, \boldsymbol{v}_{h}\right),
\end{aligned}
$$

where:

$$
\begin{aligned}
& \Sigma_{p}\left(p, \boldsymbol{v}_{h}\right)=-\sum_{e \in \varepsilon_{h}} \int_{e} p\left[\boldsymbol{v}_{h} \cdot \boldsymbol{n}\right] d s \\
& \Sigma_{u}\left(\boldsymbol{u}, \boldsymbol{v}_{h}\right)=2 \eta \sum_{e \in \varepsilon_{h}} \int_{e} \underline{D}(\boldsymbol{u}) \cdot \boldsymbol{n}\left[\boldsymbol{v}_{h}\right] d s, \\
& \varepsilon\left(\boldsymbol{u}, \boldsymbol{v}_{h}\right)=(2-\delta) \eta \sum_{K \in \mathcal{K}_{h}} \int_{K}\left(\pi_{0}^{K} \underline{D}(\boldsymbol{u})-\underline{D}(\boldsymbol{u})\right): \underline{D}\left(\boldsymbol{v}_{h}\right) d x .
\end{aligned}
$$

Then we classically show:

$$
\begin{array}{r}
\alpha\left[\left[\boldsymbol{u}_{h}-\boldsymbol{I}_{h} \boldsymbol{u}\right]\right] \leq c h|\boldsymbol{u}|_{2, \Omega}+c h|p|_{1, \Omega} \\
+\quad \sup _{\boldsymbol{w}_{h} \in \boldsymbol{V}_{h}^{\mathbf{o}}} \frac{\left|\Sigma_{p}\left(p, \boldsymbol{w}_{h}\right)-\Sigma_{u}\left(\boldsymbol{u}, \boldsymbol{w}_{h}\right)-\varepsilon\left(\boldsymbol{u}, \boldsymbol{w}_{h}\right)\right|}{\left[\left[\boldsymbol{w}_{h}\right]\right]} .
\end{array}
$$

The first two terms of the consistency error, which take into account the nonconformity of the method, are bounded similarly to [14], thanks to the Korn inequality (4): 
$\Sigma_{p}\left(p, \boldsymbol{w}_{h}\right)+\Sigma_{u}\left(\boldsymbol{u}, \boldsymbol{w}_{h}\right) \leq \operatorname{ch}\left(\frac{1}{\sqrt{\eta}}|p|_{1 \Omega}+\sqrt{\eta}|\boldsymbol{u}|_{2, \Omega}\right)\left[\left[\boldsymbol{w}_{h}\right]\right]$.

Using the Cauchy-Schwarz inequality, we also obtain:

$$
\varepsilon\left(\boldsymbol{u}, \boldsymbol{w}_{h}\right) \leq \operatorname{ch} \sqrt{\eta}|\boldsymbol{u}|_{2, h}\left[\left[\boldsymbol{w}_{h}\right]\right],
$$

which allows us to conclude.

If we omit the term $R(\cdot, \cdot)$, which may seem natural at a first glance, then the corresponding two-fields formulation (3) has a unique solution but is not consistent. Indeed, the norm $[[\cdot]]$ is now replaced by:

$$
[[[\boldsymbol{v}]]]=\left(2 \eta \sum_{K \in \mathcal{K}_{h}}\left\|\pi_{0}^{K} \underline{D}(\boldsymbol{v})\right\|_{0, K}^{2}+\gamma J(\boldsymbol{v}, \boldsymbol{v})\right)^{1 / 2} .
$$

In order to bound the consistency error with respect to $[[[\cdot]]]$, one needs the following uniform estimates:

$$
\left|\boldsymbol{w}_{h}\right|_{1, h} \leq c_{1}\left[\left[\left[\boldsymbol{w}_{h}\right]\right]\right], \quad\left\|\underline{D}\left(\boldsymbol{w}_{h}\right)\right\|_{0, \Omega} \leq c_{2}\left[\left[\left[\boldsymbol{w}_{h}\right]\right]\right],
$$

which do not hold on $\boldsymbol{V}_{h}^{\mathbf{0}}$. To illustrate numerically this phenomenon, we show in Fig.1 the results obtained for $u_{2}$ and $\tau_{12}$ for the driven cavity with (Fig.1(b)) and without (Fig.1(c)) regularization. We have also considered a triangular mesh (Fig.1(a)) with a Crouzeix-Raviart (see [3]) nonconforming approximation as a reference solution. One may clearly see that the velocity given in Fig.1(c) is not correct. Moreover, $\tau_{12}$ computed without $R(\cdot, \cdot)$ is constant (and hence not represented in Fig.1(c)).

\section{NUMERICAL RESULTS}

We have implemented the previous numerical scheme in the $\mathrm{C}++$ library Concha ${ }^{1}$. In order to validate the approximation, we first study the behavior of the errors with respect to mesh refinement. Then we consider a popular benchmark problem, the flow past a cylinder and we compare our results with those obtained with the commercial code Polyflow ${ }^{\circledR}$. Comparisons with drag values found in the literature for the Oldroyd-B model are also carried out. Finally, we show that simulations for high Weissenberg numbers give physically acceptable results and briefly justify it. We have taken in all the tests $\gamma=10$ and $\delta=2$.

\subsection{Convergence with respect to mesh refinement}

No analytical exact solution of the Giesekus model is known, hence we consider a right-hand side such that the solution on $\Omega=[-1,1] \times[-1,1]$ with Dirichlet conditions is:

$$
\begin{aligned}
& \boldsymbol{u}(x, y)=\left(\begin{array}{c}
1+x+2 x^{2}+2 y^{2}+x y \\
1-x+2 x^{2}-0.5 y^{2}-4 x y
\end{array}\right), \\
& p(x, y)=x-y, \\
& \underline{\tau}(x, y)=\left(\begin{array}{cc}
2 x+3 y & 1+3 x+4 y \\
1+3 x+4 y & 2+4 x+5 y
\end{array}\right) .
\end{aligned}
$$

\footnotetext{
${ }^{1}$ http://sites.google.com/site/conchapau
}

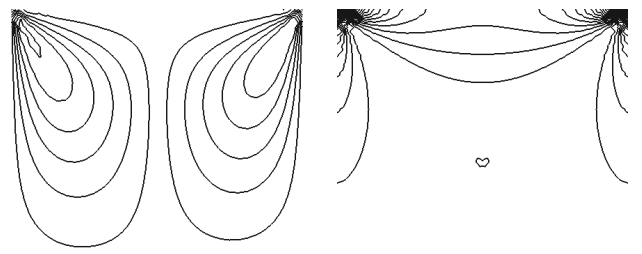

(a) Triangular mesh : $u_{2}$ and $\tau_{12}$

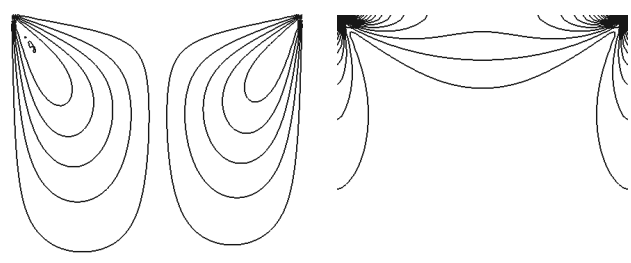

(b) Quad. mesh with $R(\cdot, \cdot): u_{2}$ and $\tau_{12}$

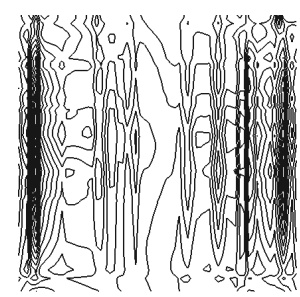

(c) Quad. mesh without $R(\cdot, \cdot): u_{2}$

Figure 1: Driven cavity : influence of $R(\cdot, \cdot)$

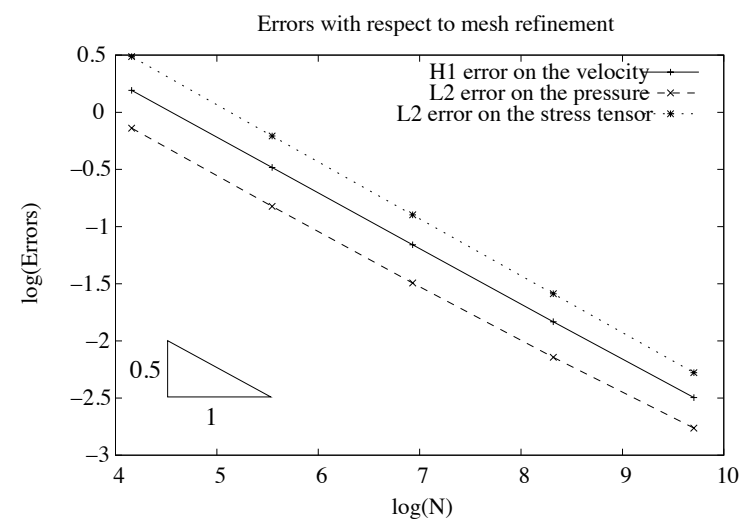

Figure 2: Convergence with respect to mesh refinement

We have shown in Fig. 2 the $H^{1}(\Omega)$-error of the velocity and the $L^{2}(\Omega)$-errors of the pressure and of the stress tensor in $\log$-log scale, for $\lambda=0.1$ and $\eta=1$. We get the expected convergence rate $\mathcal{O}\left(N^{-1 / 2}\right)$ for the three unknowns.

\subsection{Flow past a cylinder}

We consider a popular benchmark in the computational rheology community, a $2 \mathrm{D}$ flow past a cylinder. Similar results were obtained on other test-cases like $4: 1$ and $4: 1: 4$ 
contractions. The geometry is described in Fig.3 where $R$, the radius of the cylinder, is equal to $1 \mathrm{~m}$. We impose the same inflow conditions as in [4], in particular a parabolic velocity profile with $u_{\text {mean }}=1 \mathrm{~m} / \mathrm{s}$. On the outflow we impose a homogeneous Neumann condition, and on the other boundaries, including the cylinder, no-slip conditions.

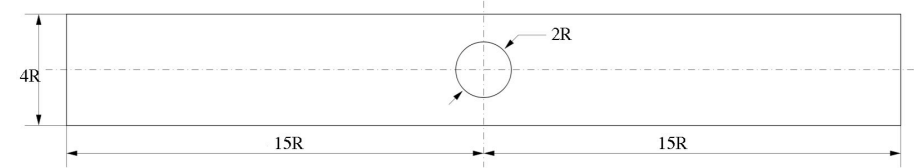

Figure 3: Geometry for a flow past a cylinder

\subsubsection{Drag comparisons for the Oldroyd-B model}

We now carry out some comparisons with results of the literature, in particular with [12], [8], [5] and [4] where reference drag values for the following Oldroyd-B model are given:

$$
\left\{\begin{aligned}
-2 \eta_{n} \operatorname{div}(\underline{D}(\boldsymbol{v}))+\nabla p & =\operatorname{div} \underline{\tau}_{p} \\
\nabla \cdot \boldsymbol{v} & =0 \\
\underline{\tau}_{p}+\lambda \underline{\tau}_{p} & =2 \eta_{p} \underline{D}(\boldsymbol{v}) .
\end{aligned}\right.
$$

As in the cited papers, we take $\eta=\eta_{n}+\eta_{p}=1, \eta_{p}=0.41$ and the Weissenberg number defined by $W e^{*}=\frac{\lambda u_{\text {mean }}}{R}$, with $u_{\text {mean }}$ the inflow mean velocity. The drag along the cylinder $\Gamma_{c}$ is given by the relation $\mathcal{C}=\int_{\Gamma_{c}}(1,0)^{T} \cdot \underline{\Pi} \boldsymbol{n} d s$, with $\underline{\Pi}=\underline{\tau}_{p}-p \underline{I}+2 \eta_{n} \underline{D}(\boldsymbol{u})$ the total stress tensor. We have implemented the same numerical scheme as for the Giesekus model. In order to obtain accurate drag values, we have solved the linear system on a very fine mesh thanks to a multigrid method based on Vanka's smoother.

The values of $\mathcal{C}$ on successive meshes, for $\lambda=0.6$, are given in Tab.1. The linear convergence obtained leads to more accurate extrapolated values $\mathcal{C}^{*}$. $n_{N}$ denotes the number of Newton iterates whereas $n_{M}$ is the sum of the multigrid iterates. One may see in Tab.2 that the drag

\begin{tabular}{|c|c|c|c|c|c|}
\hline$N$ & $n_{N}$ & $n_{M}$ & $\mathcal{C}$ & $\Delta \mathcal{C}$ & $\mathcal{C}^{*}$ \\
\hline 1024 & 7 & 19 & 118.081 & - & - \\
4096 & 6 & 12 & 118.421 & 0.340 & - \\
16384 & 6 & 18 & 118.349 & 0.072 & - \\
65536 & 6 & 24 & 118.085 & 0.264 & 117.821 \\
262144 & 5 & 20 & 117.936 & 0.149 & 117.787 \\
1048576 & 5 & 31 & 117.858 & 0.078 & 117.780 \\
\hline
\end{tabular}

Table 1: Drag values for $\lambda=0.6$

values $\mathcal{C}^{*}$ obtained with Concha on a mesh consisting of 1048576 elements for different $\lambda$ are quite close to those of the literature, in particular with [8] and [4].

\begin{tabular}{|c||c|c|c|c|}
\hline$\lambda$ & 0.0 & 0.3 & 0.6 & 0.7 \\
\hline \hline Concha & 132.357 & 123.190 & 117.780 & 117.321 \\
Ref. [12] & 132.357 & - & 117.775 & - \\
Ref. [8] & 132.358 & 123.193 & 117.792 & 117.29 \\
Ref. [5] & 132.33 & 123.41 & - & - \\
Ref. [4] & - & 123.194 & 117.779 & 117.321 \\
\hline
\end{tabular}

Table 2: Comparison of drag values with the literature

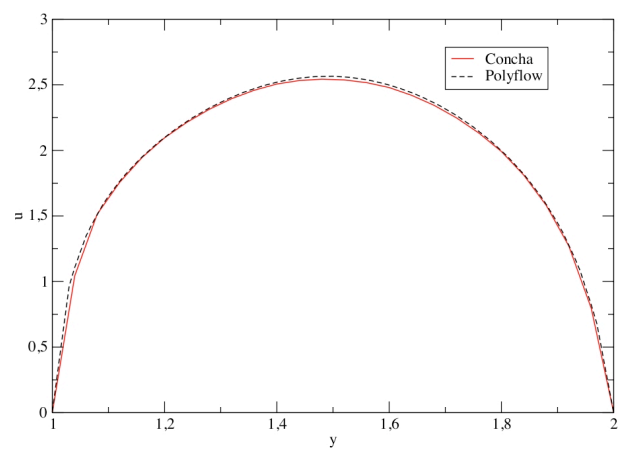

Figure 4: Velocity on a vertical axis

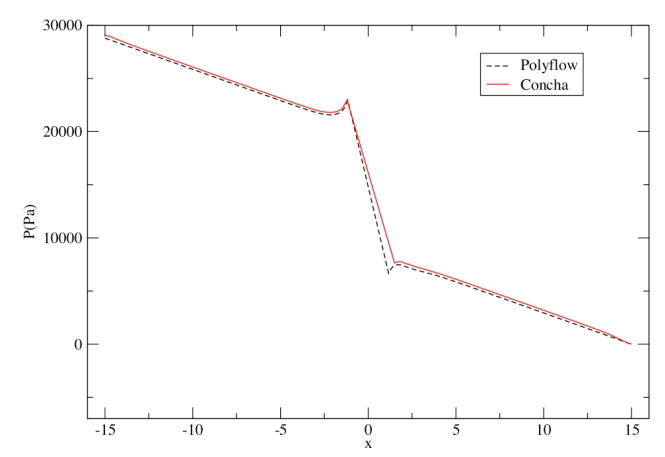

Figure 5: Pressure on the symmetry axis

\subsubsection{Comparison with Polyflow ${ }^{\circledR}$}

From now on, we simulate the Giesekus model for which we compute the Weissenberg number by the relation:

$$
W e=\lambda \dot{\gamma}=12 \lambda \bar{u}
$$

where $\bar{u}$ is the mean velocity in the thin channel. Note that the shear-rate $\dot{\gamma}$ is computed for an equivalent Newtonian liquid. We take $\eta=1000$ Pa.s and $\rho=1000 \mathrm{~kg} / \mathrm{m}^{3}$.

We compare in Fig.4 and Fig.5 our results with those obtained with Polyflow ${ }^{\circledR}$ which is the most popular commercial code for the simulation of polymer flows. The discretisation used in Polyflow ${ }^{\circledR}$ is $Q_{2}$-continuous elements for the velocity, $Q_{1}$-continuous for the pressure and EVSS method with streamline-upwind for the stress tensor.

In Fig.4, the velocity profiles along the vertical axis passing through the centre of the cylinder, in the half domain, are shown. In Fig.5 we present the pressures obtained along the horizontal symmetry axis of the channel. 
These results are obtained for $W e=6$ since for higher values, Polyflow ${ }^{\circledR}$ has difficulties to converge. One observes a good agreement between the two approaches, the differences can be explained by the different meshes used.

\subsubsection{Simulations for high Weissenberg numbers}

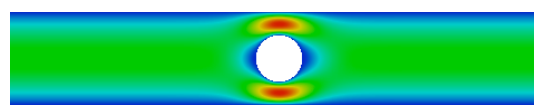

(a) $W e=0$

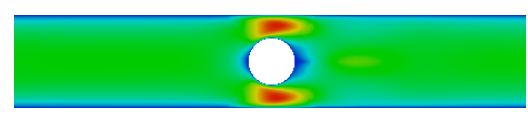

(b) $W e=24$

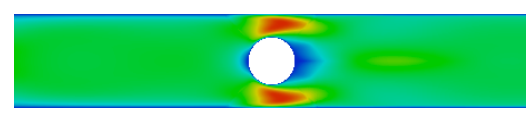

(c) $W e=60$

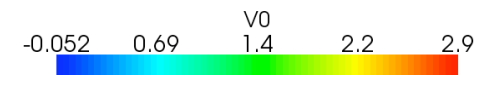

Figure 6: First componenent of the velocity

The loss of convergence of the algorithms for high Weissenberg numbers is a major issue in computational rheology and is associated with the loss of the positivity of the so-called conformation tensor at the discrete level. $\mathrm{Nu}$ merical schemes preserving this property yield energy estimates and are more stable. One approach to preserve the discrete positivity is to re-write the constitutive equation in terms of the logarithm of the conformation tensor, see for instance [8] and [4]. At our knowledge, no convergence analysis is known for this change of variable.

Another approach, introduced by Lee and $\mathrm{Xu}$ in [9], uses the similarity between constitutive laws of viscoelastic flows and Riccati equations. Their result relies on the characteristics method. It is shown in [1] that our discretisation allows to write the discrete Giesekus law as an algebraic Riccati equation, ensuring the positivity of the discrete conformation tensor under mild conditions.

In this paragraph we illustrate the stability of the scheme for high Weissenberg numbers. Moreover, the simulations exhibit the specific behavior of polymers flows which are related to their elastic character and which increase with the relaxation time. In Fig.6 one can observe two swellings after the cylinder, explained by the emergence of important normal stresses above and below the cylinder, and also by the memory effect.

Finally, we show in Fig.7 the velocity profiles along the same axis as in Fig.4 for a Newtonian liquid $(W e=0)$ and for $W e=48$. The asymmetric velocity profile is typical for a polymeric liquid and is due to the memory effect.

In conclusion, we have proposed a nonconforming approximation of a realistic polymer model, shown its optimality for the Stokes equations and validated it by means

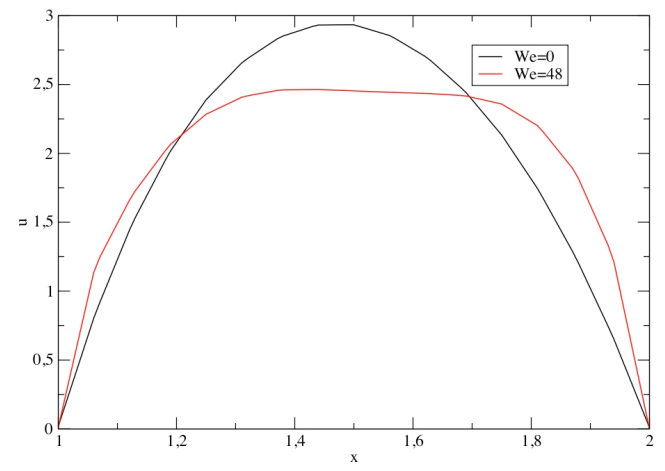

Figure 7: Velocity profiles for $W e=0$ and $W e=48$

of several comparisons . We have illustrated its robustness for large Weissenberg numbers which can be explained by the positivity of the conformation tensor, achieved without a log transformation.

\section{References}

[1] R. Becker and D. Capatina. Finite element discretization of the Giesekus model for polymer flows. Proceeding of ENUMATH'09, Springer Verlag, 2010.

[2] S. C. Brenner. Korn's inequalities for piecewise $H^{1}$ vector fields. Math. Comp., 73(247):1067-1087, 2004.

[3] M. Crouzeix and P.-A. Raviart. Conforming and nonconforming finite element methods for solving the stationary Stokes equations. I. RAIRO, 7(R-3):33-75, 1973.

[4] H. Damanik, J. Hron, A. Ouazzi, and S. Turek. A monolithic FEM approach for the $\log$-conformation reformulation (lcr) of viscoelastic flow problems. J. Non-Newtonian Fluid Mech., 2010. to appear.

[5] J. Étienne, E. J. Hinch, and J. Li. A Lagrangian-Eulerian approach for the numerical simulation of free-surface flow of a viscoelastic material. Journal of Non-Newtonian Fluid Mechanics, 136:157, 2006

[6] H. Giesekus. A simple constitutive equation for polymer fluids based on the concept of deformation-dependent tensorial mobility. J.Non-Newtonian Fluid Mechanics, 11:69-109, 1982.

[7] R. Guénette and M. Fortin. A new mixed finite element method for computing viscoelastic flows. J.Non-Newtonian Fluid Mech., $60: 27,1995$.

[8] M. A. Hulsen, R. Fattal, and R. Kupferman. Flow of viscoelastic fluids past a cylinder at high Weissenberg number: Stabilized simulations using matrix logarithms. Journal of NonNewtonian Fluid Mechanics, 127:27, 2005.

[9] Y.-J. Lee and J. Xu. New formulations, positivity preserving discretizations and stability analysis for non-Newtonian flow models. Comp. Methods Appl. Mech. Engrg., 195 1180, 2006.

[10] P. Lesaint and P. A. Raviart. On a finite element method for solving the neutron transport equation. Mathematical Aspects of Finite Element Methods in PDE's. C.A. de Boor (Ed.), Academic Press, 1974.

[11] K.-A. Mardal and R. Winther. An observation on Korn's inequality for nonconforming finite element methods. Math. Comp., 75(253):1-6, 2005.

[12] R. G. Owens, C. Chauvire and T. N. Philips A locally-upwinded spectral technique (LUST) for viscoelastic flows. Journal of Non-Newtonian Fluid Mechanics, 108:49, 2002.

[13] R. G. Owens and T. N. Phillips. Computational rheology. Imperial College Press, London, 2002.

[14] R. Rannacher and S. Turek. Simple nonconforming quadrilateral Stokes element. Numer. Methods Partial Differential Equations, 8(2) $97,1992$. 